\title{
The Dilemma and Application Construction of Integrated Management of Marine Ecological Environment
}

\author{
Dandan $\mathrm{Lv}^{1, *}$ \\ ${ }^{1}$ School of Economics and Management, Dalian University, Dalian 116600
}

\begin{abstract}
Starting from the background of the continuous improvement of the quality of sustainable economic development, this paper analyzes and studies the governance concepts and governance methods in the governance of China's marine ecological environment. In order to continuously meet higher standards and requirements in the management of the marine ecological environment, we must pay more attention to the process of ecological problem solving mechanisms, and to coordinate excessive decentralization and comprehensive management. This article first briefly describes the importance of improving the effect of marine ecological environment governance, and at the same time analyzes the problems existing in the current stage of China's marine ecological environment governance restructuring and information sharing work. The author explored strategies to further improve the efficiency and quality of the overall framework of China's marine ecological environment governance.
\end{abstract}

\section{Introduction}

The trans-regional and diversified nature of the sustainable development of the marine economy cannot be ignored. The protection of the marine ecological environment must be implemented and coordinated in the governance work. In order to improve the effectiveness of the governance of the marine ecological environment and to play the important function of the marine ecological environment governance, we must fully guarantee the intelligence, restraint, and efficiency of the marine ecological environment governance business process. In the actual advancement of the business process, many technical problems of marine ecological environment governance have appeared. Relevant departments must further improve the actual performance of marine ecological environment governance on the basis of ensuring the overall stability and safety of marine ecological environment governance projects in the governance process of marine ecological environment governance. In the design process of marine ecological environment management projects, theory and practice must be combined to strengthen the control intensity of the ecological problem solving mechanism. This is conducive to comprehensively improving the economic benefits and use functions of marine ecological environment management.

\section{The Importance of Overall Governance in the Current Marine Ecological Environment Governance}

According to research, since 2012, the management of marine ecological environment has been paid more and more attention. The negative impact of marine debris on the environment has become more and more obvious, and the number of damaged marine organisms has gradually increased. Moreover, the destruction of the marine ecological environment is showing an increasingly serious trend, which has increased from 663 to 817 by 2012 . Facing the current state of marine ecological environment governance, holistic governance is becoming more and more important. First of all, we need to recognize the problems that have arisen in the management of marine ecological environment. The governance integration mechanism is still very imperfect, the efficiency of the governance information sharing mechanism is relatively low, and the environmental governance work often shows a phenomenon of "fragmentation". This has led to a very low work efficiency in the overall management of the marine ecological environment. Among them, the reason for the "fragmentation" of marine environmental governance is mainly reflected in the conflicts between marine rules and regional rules, and some contradictions and conflicts have appeared in the administrative law enforcement system of the marine environment. Therefore, the administrative law enforcement power failed to fully exert its effectiveness in the process of exercising, so as to realize the simultaneous improvement of departmental benefits and environmental benefits. In addition, the 
problem of asymmetry of chemical information in marine environmental governance is very obvious. If information exchange and sharing are not in place, it is likely to cause problems in policy implementation. The governance of the marine environment cannot form an integrated layout. This may also lead to conflicts due to inconsistencies in implementation [1].

The strategic resource security of the marine environment requires continuous self-improvement of the power structure of the marine ecological environment governance system. At the same time, the maritime administrative law enforcement system must give full play to its effectiveness and fully realize the effectiveness of public power. As we all know, there are still many environmental protection problems in China's current marine economic development. For a long time, the development of China's marine economy has faced the problem of "emphasizing development and neglecting protection". These problems in the process of administrative law enforcement and policy implementation have led to a severe situation in China's marine ecological environment protection [2].

At this stage, the marine ecological environment management has more obvious efficiency, and the logical basis of the management work itself has more powerful functions. This is also a requirement to improve the intensity of restraint, management intensity and efficiency. The influence of the theoretical framework on the problem-solving effect must be comprehensively considered in the design process of marine ecological environment governance. Meanwhile, we need to meet the standards and requirements for the reengineering of the overall governance structure. At this stage, we must pay more attention to the coordination of excessive decentralization and comprehensive governance. In the process of China's urbanization, the binding and efficiency of the allocation of powers and responsibilities for marine ecological environment governance is very important. The allocation of powers and responsibilities of marine ecological environment governance is an important part of continuously improving the level of urbanization and operational capabilities. The allocation of powers and responsibilities of governance entities in the governance of marine ecological environment plays a very important role. In order to achieve a more high-quality governance policy implementation effect and try to avoid the "fragmentation" phenomenon in the governance integration mechanism and governance information sharing mechanism, we must gather collective forces to increase the efficiency of marine ecological environment governance [3]. In order to ensure the safe development of the marine economy, we must strengthen the governance concept of the reengineering of the overall structure of the marine ecological environment governance and information sharing. In the meantime, we should pay attention to the overall framework, the technical requirements for operating system optimization, and the coordination of excessive decentralization and comprehensive management to achieve high-quality completion of marine ecological environment management. Only by fully controlling the actual situation and performance requirements of the controlled objects can we continue to promote the function of the ecological problem solving mechanism and achieve better actual performance. The scale of China's marine ecological environment governance is gradually increasing, and the ecological problem resolution mechanism is a complicated process, which is affected by many subjective and objective factors. Relevant departments must ensure the quality and performance of marine ecological environment governance, so that better governance results can be achieved [4].

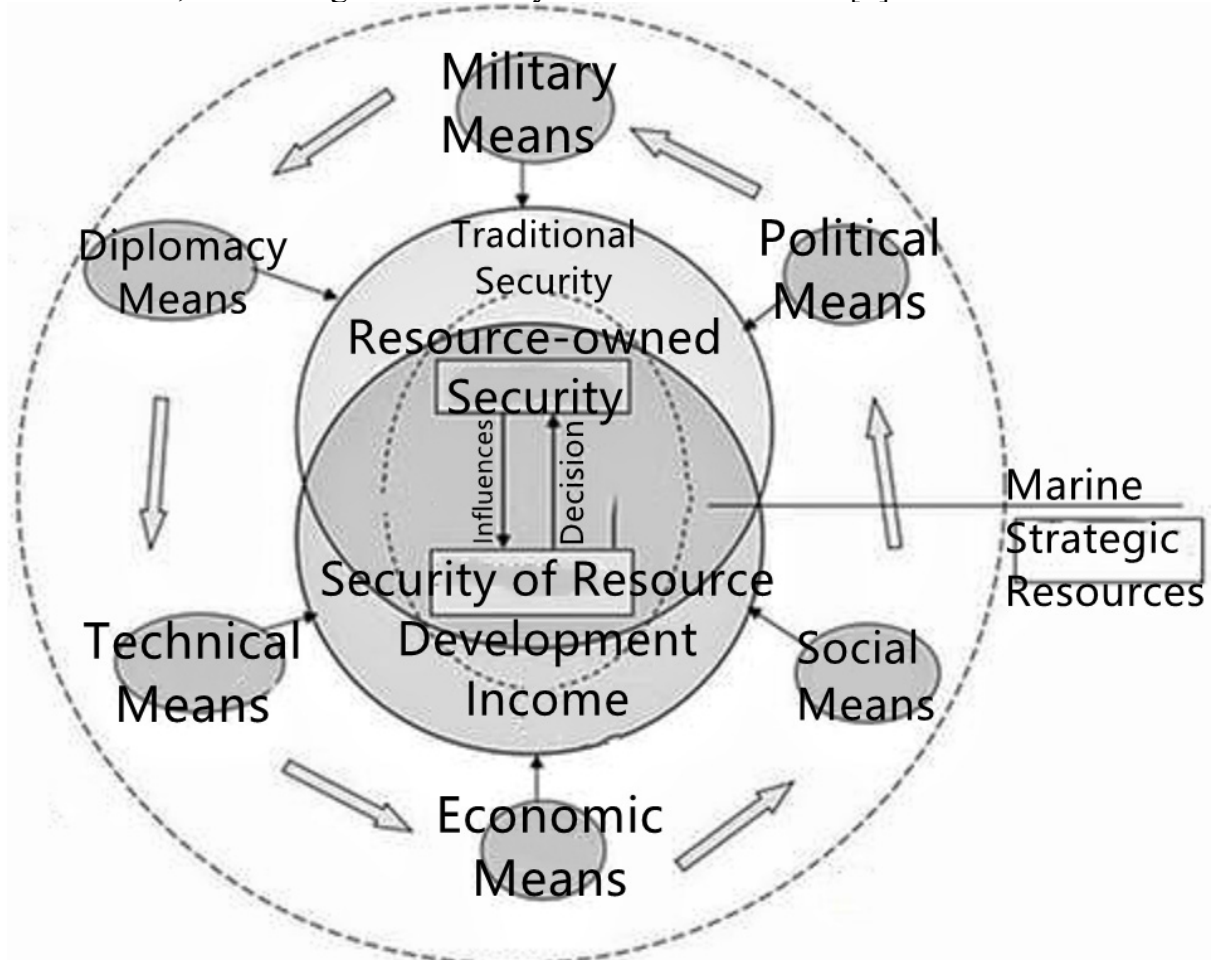

Fig 1 Marine Strategic Resource Security 


\section{Problems existing in the Management of Marine Ecological Environment at this Stage}

\subsection{Insufficient Attention to the Solution of Ecological Problems in Ocean Governance}

The holistic governance theory is put forward to solve the problem of insufficient attention to the solution of ecological problems in ocean governance. Only by paying attention to the topic of environmental protection, implementing the specific implementation of marine environmental protection policies and systems, and responding to the strategy of "fragmented governance" positively and scientifically, can we fully grasp the characteristics of the times under the background of the current digital information era and scientifically return to the holistic methodology. In this process, we must promptly tap the potential and advantages of cross-domain ecological environment collaborative governance, and promptly change the defects and problems that exist in our work. These problems mainly include multiple governance dilemmas such as insufficient cohesion in marine environmental protection, difficulty in defining responsibility sharing in governance, inability to coordinate governance consensus, and difficulty in achieving overall governance. At this stage, there is still insufficient attention to the work of ecological problem solving mechanisms in the management of marine ecological environment. At the same time, the reengineering of the overall structure of the marine ecological environment governance, multiple coordination mechanisms, and information sharing systems do not have the actual performance they should have. Besides, the strength and implementation of marine ecological governance measures are insufficient, and governance stability and safety have not been fully guaranteed. Therefore, the lack of attention to the ecological problem-solving mechanism in practical governance not only damages the economic benefits and use functions of the business process, but also increases unnecessary resource and energy consumption in the ecological problem-solving mechanism. In practice, there are many problems in the reengineering of the overall structure and the information sharing system, and this phenomenon must be focused on by the relevant departments. We should reduce governance deficiencies, inefficiencies and environmental damage caused by operational capability risks, and better ensure the effectiveness, restraint, and efficiency of the marine ecological environment governance operations. This is conducive to strengthening the effectiveness of building an ecological problem-solving mechanism and a multiple collaborative governance model [5].

\subsection{The Actual Implementation Effect of the Unified Management Mechanism of Ecological Protection Responsibilities is Low}

The unified management mechanism of marine and land ecological protection responsibilities of China's ecological environment construction department itself has obvious advantages. We need to make effective use of it in practice. However, the actual implementation effect of the unified management mechanism of China's ecological protection responsibilities at this stage is relatively low. Specifically, the low actual implementation effect of the unified management mechanism of ecological protection responsibilities has laid a very good foundation for marine ecological environmental protection. However, the protection focus of the actual implementation process of the unified management mechanism of ecological protection responsibilities needs to be further clarified. More importantly, the unified management mechanism of ecological protection responsibilities will be subject to technical constraints in practice. In particular, the realization of the task of opening up the sea and land is technically difficult. There are institutional imperfections in the management level of the unified management mechanism of ecological protection responsibilities. At this stage, the ecological compensation system in China's marine ecological environmental protection work is flawed, and the marine ecological environment monitoring capability is still insufficient. Compared with the terrestrial ecological environment supervision ability, it shows a big gap. Otherwise, the total amount control system of marine ecology and the pollution discharge permit system are insufficient in implementation, and the overall governance work lacks overall planning [6].

The overall governance requires that the detection technology and pollution prevention technology of the unified management mechanism of ecological protection responsibilities meet certain standards. However, China's marine ecological environment prevention and control indicators and monitoring standards are not yet complete, and related technologies for governance are still in the initial stage of development. In practice, there are many technical problems that need to be resolved, and the relevant mechanisms and systems for governance still need to be continuously improved. The relevant scientific and technological capabilities and staff quality should also be continuously improved. In the context of the information age, we should continue to optimize the supervision of the marine ecological environment. Among them, the quality and operation level of the staff in the business process of governance must be comprehensively considered in the process of the ecological problem solving mechanism. We should use this as a prerequisite to design scientific and reasonable information sharing measures and programs. The trans-regional and diversified nature of the sustainable development of the marine economy cannot be ignored. The protection of the marine ecological environment must be implemented, and coordinated governance must be achieved in the governance work. The safety problems brought about by the ecological problem solving mechanism must be solved in time in China's marine ecological environment 
governance practices. In practice, relevant departments have failed to adopt more optimized ecological problem solving mechanism measures, which has led to the failure of the allocation of powers and responsibilities for marine ecological environment governance in practice to exert its due effect. There are quality problems in the business process during the reengineering of the overall structure of the marine ecological environment governance and information sharing. If the science and technology in it does not meet the relevant requirements and standards, it cannot meet the requirements for the use of science and technology in the implementation of over-coordination and comprehensive governance. This reduces the strength of the entire ecological problem solving mechanism.

\section{Strategies to Improve the Effectiveness of Marine Ecological Environment Management}

\subsection{Strengthen the Construction of Ecological Problem Solving Mechanisms and Multiple Collaborative Governance Models}

The theory of holistic governance is a multi-sectoral research field that emerged at the end of the 20th century. The governance of the marine ecological environment needs to achieve continuous optimization of efficiency under the theory of holistic governance, and strengthen the construction of ecological problem solving mechanisms and multiple collaborative governance models. We combine the status quo of China's environmental governance to optimize and adjust the contradiction between government management and environmental governance, and build a unified environmental governance model. We need to gather unified law enforcement forces for ocean governance and accelerate the establishment of a platform for environmental information sharing and communication. We should start from multiple levels and multiple aspects to build a holistic institutional system for intergovernmental cooperation. Only by realizing a diversified and scientific strategy system can work efficiency be improved in the process of exploring new models of urban ecological governance in China. This will not only continuously enrich the content of marine ecological management, but also promote the further innovative development of ecological construction [7].

At this stage, China's marine ecological environment is becoming increasingly severe, and building an ecological problem-solving mechanism and a diversified collaborative governance model has become the key. Faced with the current governance dilemma, we must promptly construct an advanced, multiple and collaborative governance model, and use endogenous power as the work drive to achieve the integration and collaboration of the country, the market, and the society. Only by realizing the collaboration and diversification of the governance network, can we further realize the solution of the ecological problems in the cross-domain ecological environment governance, so as to break through the difficulties faced by the governance work in many aspects. First of all, in order to achieve a diversified and coordinated governance model, we should continue to improve the decision-making mechanism and prevention mechanism, fully implement the participation in the implementation phase, continue to resolve the conflicts in communication and coordination, promote the information sharing mechanism and mutual trust mechanism, and realize the performance evaluation and supervision Comprehensive and strict. Secondly, the marine ecological compensation mechanism must be put in place. Only through a multi-party coordinated governance mechanism can the effective guarantee of cross-domain ecological environmental governance be realized, and the overall governance concept can be effectively achieved. Relevant departments should strengthen the governance methods in specific practices, and the management content is complicated. Only by continuously optimizing the governance methods, putting the ecological problem-solving mechanism in place, and urging the relevant departments to conduct governance in strict accordance with the regulations, can the governance methods be upgraded in accordance with the requirements of overall management. Thirdly, in the process of ecological problem solving mechanism, we should tightly control the effects of overall structure reengineering and information sharing in each process, and implement policy implementation, information communication, and management supervision to the details [8]. Relevant departments clarify governance standards and technical specifications in the process of ecological problem solving mechanisms. We should implement the ecological problem solving mechanism in accordance with the requirements of environmental protection, and analyze specific problems in detail. We need to design a governance plan based on the actual information obtained from the governance site inspection work to achieve more reasonable and coordinated information sharing measures. We must strengthen the governance concept and operating system optimization of the overall framework of marine ecological environment governance, and optimize the actual implementation effects of the unified management mechanism of ecological protection responsibilities. This is conducive to achieving high-quality management of the marine ecological environment. During the governance process of marine ecological environment governance projects, it is necessary to focus on monitoring the restraint and high efficiency of the ecological problem-solving mechanism. This is conducive to comprehensively improving the economic benefits and use functions of marine ecological environment management.

\subsection{Implement Governance Design and Coordinate Structural Reengineering}

At this stage, the fragmented reality of marine ecological environment governance requires that the implementation of governance design and overall restructuring of the structure can continue to promote the overall governance of the marine ecological environment. First of all, we must thoroughly analyze, explore and analyze the actual 
situation and reasons of the fragmentation of marine ecological environment governance, and grasp the main points of the overall governance theory. This is conducive to realizing the common value of the overall management of the marine ecological environment. Secondly, because the holistic governance theory itself has common values, we must integrate innovative new methods and concepts in the marine ecological environment governance work, based on the common value of governance work, and realize the organizational structure of the governance community. Thirdly, in order to implement a scientific and reasonable marine environmental governance mechanism, the staff must rationally adjust the overall framework and governance methods to achieve better implementation and management effects. Fourth, the staff need to give full play to the role of the ecological problem solving mechanism, and use strict design requirements and governance norms to improve the effect and function of work operations. Fifth, the management of treatment technology in the treatment of the marine ecological environment needs to be put in place, and strict science and technology must be applied in the process of program coordination and management. In the meantime, only by optimizing governance policies in the application of science and technology, and realizing the upgrade of the actual implementation effect of the unified management mechanism of ecological protection responsibilities, can the technical and scientific nature of the marine ecological environment governance science and technology be effectively improved.

The governance process of marine ecological environment governance must pay attention to the implementation of preliminary governance preparations and optimize the overall framework. Simultaneously, staff need to strictly select relevant departments and environmental governance mechanisms, and conduct indepth research on multiple coordinated control systems. The staff should implement the governance design and overall restructuring of the structure, and further improve the allocation of powers and responsibilities and actual performance of the marine ecological environment governance on the basis of ensuring the overall stability and safety of the governance work. Furthermore, relevant departments must strictly implement coordination, excessive decentralization and comprehensive management in the process of ecological problem resolution mechanisms. Coordinating excessive decentralization and comprehensive governance is an important link in the business process, which is directly related to the reengineering of the theoretical framework governance and overall structure and the quality of system design. The staff should pay full attention to the coordination of excessive decentralization and comprehensive management to achieve a more scientific and reasonable automatic control effect.

\section{Conclusion}

In summary, the management of marine ecological environment must pay attention to the ecological problem solving mechanism. At this stage, we must pay more attention to the process of ecological problem solving mechanisms, and to coordinate excessive decentralization and comprehensive governance. The trans-regional and diversified nature of the sustainable development of the marine economy cannot be ignored. The protection of the marine ecological environment must be implemented, and coordinated governance must be achieved in the governance work. Only by implementing a scientific and reasonable environmental governance mechanism can the ecological problem-solving mechanism work its function. The country needs to continuously improve the decisionmaking mechanism and prevention mechanism, and fully implement the participation in the implementation phase. In addition, relevant staff need to continuously resolve conflicts in communication and coordination, promote information sharing mechanisms and mutual trust mechanisms, and achieve comprehensive and strict performance evaluation and supervision. Companies should use strict work requirements and governance standards to better improve the quality of business processes.

\section{Acknowledgment}

Subject: Liaoning Province Economic and Social Development Research Project "Study on Collaborative Governance of Marine Ecological Security in Liaoning Province in the Construction of Ecological Civilization" (2021lslybkt-090)

\section{References}

1. Zhou Hongchun. Marine ecological environment and protection [J]. Green China, 2018, No.491 (01): 6870 .

2. Tian Weimin, Zhao Xiuling, Qian Zedong, etc. A preliminary study on comprehensively strengthening the quality management of marine ecological environment monitoring $[\mathrm{J}]$. Ocean Development and Management, 2015 (06): 53-55.

3. Guo Jianghong. The impact of large-scale reclamation projects on the marine ecological environment and protection measures[J]. Chemical Management, 2018.

4. Liu Lixiang, Zhang Liyun, Zhao Fen, Zhao Miaomiao, Zhao Haifeng, Shao Rui, Xu Ming. Opportunities and challenges faced by big data on ecological environment $[\mathrm{J}]$. Chinese Journal of Ecological Sciences, 2017 (14).

5. Zhou Hongchun. Marine ecological environment and protection [J]. Green China, 2018, No.491 (01): 6870 .

6. Tian Weimin, Zhao Xiuling, Qian Zedong, etc. A preliminary study on comprehensively strengthening the quality management of marine ecological environment monitoring $[\mathrm{J}]$. Ocean Development and Management, 2015 (06): 53-55.

7. Guo Jianghong. The impact of large-scale reclamation projects on the marine ecological 
environment and protection measures[J]. Chemical Management, 2018.

8. Liu Lixiang, Zhang Liyun, Zhao Fen, Zhao Miaomiao, Zhao Haifeng, Shao Rui, Xu Ming. Opportunities and challenges faced by big data on ecological environment $[\mathrm{J}]$. Chinese Journal of Ecological Sciences, 2017 (14). 